\title{
Cochlear and Neural Delays for Coincidence Detection in Owls
}

\author{
José Luis Peña, ${ }^{1}$ Svenja Viete, ${ }^{1}$ Kazuo Funabiki, ${ }^{1}$ Kourosh Saberi, ${ }^{2}$ and Masakazu Konishi ${ }^{1}$ \\ ${ }^{1}$ Division of Biology, California Institute of Technology, Pasadena, California 91125, and 2Department of Cognitive \\ Science, University of California at Irvine, Irvine, California 92697
}

The auditory system uses delay lines and coincidence detection to measure the interaural time difference (ITD). Both axons and the cochlea could provide such delays. The stereausis theory assumes that differences in wave propagation time along the basilar membrane can provide the necessary delays, if the coincidence detectors receive input from fibers innervating different loci on the left and right basilar membranes. If this hypothesis were true, the left and right inputs to coincidence detectors should differ in their frequency tuning. The owl's nucleus laminaris contains coincidence detector neurons that receive input from the left and right cochlear nuclei. Monaural frequency-tuning curves of nucleus laminaris neurons showed small interaural differences. In addition, their preferred ITDs were not correlated with the interaural frequency mismatches. Instead, the preferred ITD of the neuron agrees with that predicted from the distribution of axonal delays. Thus, there is no need to invoke mechanisms other than neural delays to explain the detection of ITDs by the barn owl's laminaris neurons.

Key words: owl; nucleus laminaris; frequency tuning; coincidence detection; delay lines; sound localization; stereausis
The auditory systems of birds and mammals use coincidence detector neurons and delay lines to measure interaural time differences (ITDs). Both the coincidence detectors and axonal delay lines are known in these animals, although the evidence for axonal delay lines in mammals is anatomical (Carr and Konishi, 1990; Yin and Chan, 1990; Smith et al., 1992). The use of axons as delay lines resembles the model put forth by Jeffress (1948) (Fig. $1 A$ ). In the avian auditory system, axons from the cochlear nucleus magnocellularis (NM) and neurons of the nucleus laminaris $(\mathrm{NL})$ are thought to constitute the delay lines and coincidence detectors, respectively (Sullivan and Konishi, 1984; Young and Rubel, 1986; Carr and Konishi, 1988, 1990; Joseph and Hyson, 1993). Both the NM and the NL are tonotopically organized, and the processing of interaural time differences occurs in separate frequency bands (Takahashi and Konishi, 1988; Carr and Konishi, 1990).

Shamma et al. (1989) suggested that the differences in wave propagation along the cochlea could provide the delays necessary for coincidence detection, if the coincidence detectors receive input from fibers innervating different loci on the left and right basilar membranes. The propagation time of the traveling wave along the basilar membrane causes sites near the oval window [high characteristic frequency $(\mathrm{CF})]$ to respond first and regions further away to respond at later times (von Bekesy, 1960). According to this "stereausis" theory, the selectivity of a coincidence detector for ITD is determined by the temporal disparity between the left and right cochlear loci from which it receives

Received Jan. 4, 2001; revised Sept. 7, 2001; accepted Sept. 13, 2001.

This work was supported by National Institute of Neurological Disorders and Stroke Grant DC-00134 and by postdoctoral fellowships from the Deutsche Forschungsgemeinschaft (S.V.) and the Pew Latin American Fellows Program (J.L.P.). We thank Christine Köppl for generously sharing data with us; Yehuda Albeck, Jamie Mazer, Chris Malek, and Ben Arthur for their help with computer programming; Catherine Carr for reviewing an early draft of the manuscript; and Mario Ruggero for discussion about cochlear mechanisms.

Correspondence should be addressed to José Luis Peña, Division of Biology 216-76, California Institute of Technology, Pasadena, CA 91125. E-mail: jose@etho.caltech.edu.

Copyright (ㄷ) 2001 Society for Neuroscience $\quad 0270-6474 / 01 / 219455-05 \$ 15.00 / 0$ inputs. In this model, coincidence detectors that receive inputs from left and right auditory neurons tuned to the same frequency would be selective for an ITD of 0 , because their propagation delays are the same for the two sides. Information about the frequency and ITD selectivity of the coincidence detectors is necessary to test the hypothesis. The owl's nucleus laminaris is particularly useful for this purpose, because NL neurons perform coincidence detection in a much higher frequency range than neurons of the chicken's nucleus laminaris or the mammalian medial nucleus of the superior olive. Because cochlear delays show smaller changes per Hertz at higher frequencies (Köppl, 1997) (Fig. 1B), larger frequency mismatches are necessary at higher frequencies than at lower ones to measure the same ITD (Shamma et al., 1989). This paper examines whether interaural frequency-tuning mismatches and/or axonal delays are correlated with the preferred ITD of nucleus laminaris neurons.

\section{MATERIALS AND METHODS}

Data were obtained from 24 adult barn owls (Tyto alba) of both sexes. Owls were anesthetized with an intramuscular injection of ketamine hydrochloride $(25 \mathrm{mg} / \mathrm{kg}$; Ketaset, Fort Dodge Animal Health, Fort Dodge, IA) and diazepam (1.3 mg/kg; Western Medical Supply, Arcadia, CA). An adequate level of anesthesia was maintained by additional injections of ketamine. After experiments, the craniotomy for electrode insertion was covered with a plastic sheet and dental cement and the skin incision was closed. Antibiotic and a local anesthetic in sterile solution were applied to the wound. Owls were returned to their individual cages and monitored for their recovery.

We isolated and maintained single neurons by a loose patch method in which the electrode served as a suction electrode, allowing us to hold neurons for many tens of minutes (Peña et al., 1996). Neural signals received by an Axoclamp-2A amplifier (Axon Instruments, Foster City, CA) were further amplified by an AC amplifier $(200 \mu \mathrm{M})$. A spike discriminator (SD1; Tucker-Davis Technologies, Gainesville, FL) converted neural impulses into transistor-transistor logic pulses for an event timer (ET1; Tucker-Davis Technologies), which recorded the timing of the pulses. A Dimension XPS Pro200n computer (Dell Computer, Round Rock, TX) was used for both online data analysis and stimulus synthesis.

An earphone assembly consisting of a Knowles 1914 receiver, a Knowles 1743 damping device, and a Knowles 1319 microphone (Knowles Electronics, Itasca, IL) delivered sound stimuli. These compo- 
A

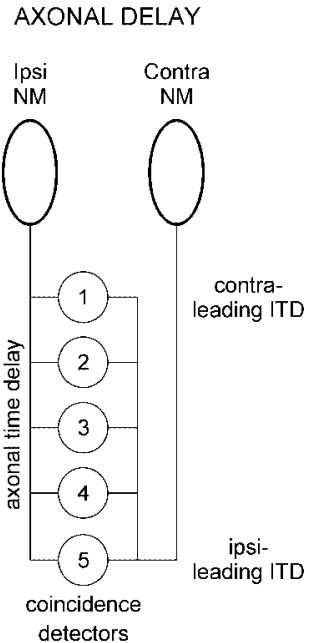

\section{COCHLEAR DELAY}

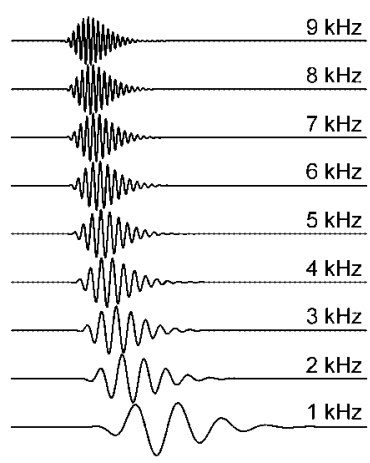

$\begin{array}{lllllllll}0 & 1 & 2 & 3 & 4 & 5 & 6 & 7 & 8\end{array}$

Figure 1. Cochlear and neural delays. $A$, Neural delays. A schematic representation of the coincidence detection circuit in the barn owl is shown. Axons from the ipsilateral NM (Ipsi NM) enter the NL on the dorsal surface; those from the contralateral NM (Contra NM) enter the $\mathrm{NL}$ on the ventral surface. The segments of these axons within the NL serve as delay lines. Binaural coincidence detectors (numbered 1-5) fire maximally when inputs from the two sides arrive simultaneously. A coincidence occurs when the sum of the acoustic delay and neural delay on one side equals that on the other side. Neuron 1 fires maximally when the sound reaches the contralateral ear first (contra-leading ITD) because a longer path delays the neural signal from the ipsilateral ear. If the sound source moves toward the ipsilateral side (ipsi-leading ITD), a coincidence occurs in neurons $2-5$ with shorter axonal paths. This array of delays forms a map of the ITD in the dorsoventral dimension of the NL. $B$, Cochlear delays computed from frequency-dependent latencies of primary auditory fibers of barn owls (Köppl, 1997; in conjunction with the cochlear model of Carney and Yin, 1988). The cochlear delay changes as a function of BF (indicated for each plot) and is the basis for the stereausis theory (Shamma et al., 1989). Note that the change in delay is smaller for higher frequencies.

nents are encased in an aluminum cylinder that fits snugly into the owl's ear canal. The gaps between the cylinder and the ear canal were filled with silicon impression material (Gold Velvet II; Earmold and Research Laboratory, Wichita, KS). At the beginning of each experimental session, both earphone assemblies were automatically calibrated and decibel sound pressure level (SPL) values for different frequencies were stored in a computer file. The computer was programmed to equalize SPL and phase for all frequencies within the frequency range relevant to the experiment.

Tonal and broadband stimuli of $100 \mathrm{msec}$ in duration with a $5 \mathrm{msec}$ rise-fall time were presented once per second and repeated five times. ITD was varied in steps of either one-tenth of the period for tonal stimuli or $30 \mu \mathrm{sec}$ for noise stimuli. We used PA4 digital attenuators (TuckerDavis Technologies) to vary stimulus sound levels. Frequency threshold tuning curves were obtained with randomized sequences of stimulus frequencies in steps of $100 \mathrm{~Hz}$. For each frequency, the threshold was determined using an iterative procedure. This method used a binary search algorithm to most efficiently define a range of sound levels (specified by a low and high value) within which the threshold is assumed to exist. At each step, the midpoint of this range was tested and the range was narrowed to either the upper or lower half depending on whether the midpoint was below or above threshold, respectively (permutation test, $p=0.05$ ) (Siegel and Castellan, 1988). For each successive range, the new midpoint was tested and the range narrowed further until it became smaller than a predetermined size (usually $5 \mathrm{~dB}$ ). Only the upper limits of these ranges are plotted in the figures presented here. This corresponds to an upper bound of the minimum sound level that evoked a discharge rate significantly higher than spontaneous activity. Thus, $\mathrm{CF}$ is defined as the frequency for which the neuron shows the lowest threshold. $\mathrm{Q}_{10}$ was computed as the ratio between $\mathrm{CF}$ and the curve width measured at $10 \mathrm{~dB}$ above the threshold at $\mathrm{CF}$.

Isointensity frequency-tuning curves were obtained for sound levels that were 20-30 dB above threshold with randomized sequences of stimulus frequencies in steps of 100 or $200 \mathrm{~Hz}$. Frequency-tuning curves were characterized by their width at half height of tuning curve $\left(\mathrm{W}_{50}\right)$ and center frequency $\left(\mathrm{F}_{50}\right)$ or best frequency $(\mathrm{BF}) . \mathrm{W}_{50}$ is the range of frequencies over which the discharge rate of the cell was equal to $50 \%$ of the difference between the maximal discharge rate and the spontaneous level. The frequency at the center of $\mathrm{W}_{50}$ was defined as $\mathrm{F}_{50}$. We used automatic methods of determining both $\mathrm{W}_{50}$ and $\mathrm{F}_{50}$. BF is usually defined as the frequency that elicits the maximal discharge rate in an isointensity frequency-tuning curve. The interaural difference in $\mathrm{F}_{50}$ was calculated by subtracting the contralateral $F_{50}$ from the ipsilateral $F_{50}$. Assuming that spike numbers show a Poisson distribution with the measured mean and SD for each test frequency, we created 1000 frequency-tuning curves using Monte Carlo randomization procedures. The SD of $\mathrm{F}_{50}$ was obtained for the 1000 randomized versions of each curve. The $\mathrm{SD}$ of ipsilateral $\mathrm{F}_{50}$-contralateral $\mathrm{F}_{50}$ corresponds to the square root of the sum of the variances of the two sides.

Precise measurement of ITDs requires an objective method, because the peaks in ITD curves from which ITDs are determined are not points. We derived ITDs by closely fitting ITD curves to cosine functions in which peak positions could be precisely measured (Viete et al., 1997). Laminaris neurons respond to an ITD and its phase equivalents, which are ITD $\pm n T$, where $\mathrm{n}$ is an integer and T is the period of the stimulus tone. Plotting of the mean interaural phase against frequency yields a line; its slope is the frequency-independent ITD or characteristic delay (Rose et al., 1966; Yin and Kuwada, 1984). This procedure works best when ITD curves for widely different frequencies can be obtained, as in low-frequency sensitive neurons (Yin and Chan, 1990). A majority of NL neurons in barn owls have narrow tuning curves in the range of $4-9 \mathrm{kHz}$. This condition makes it difficult to select frequencies that are far apart for plotting against the mean interaural phase. Even when a straight line is obtained with two or three frequencies, it may not be statistically significant. Therefore, to discriminate between the frequency-independent ITDs and their phase equivalents, we used the simple fact that ITD curves obtained for different frequencies peak at the same ITD and not at other ITDs. This ITD is the frequency-independent ITD (referred to as best ITD below for brevity). Similarly, characteristic delays occur at peaks of ITD curves in the cat's medial superior olivary nucleus (Yin and Chan, 1990). ITD curves of NL neurons are periodic even when stimuli are broadband. This periodicity enables us to measure binaural frequency tuning. We obtained the frequency of this periodicity by the cosine method mentioned above. Recordings were made on both left and right sides of the brain, but we refer to ipsilateral and contralateral sides instead. Negative ITDs represent ipsilateral side leading.

We computed ITDs predicted from frequency mismatches by using a modified version of the Bonham and Lewis (1999) cross-correlation model. We used frequency-dependent latencies of primary auditory fibers from barn owls obtained by Köppl (1997) in the absence of data on cochlear propagation delays. The model consisted of these latencies and a set of frequency-dependent GammaTone impulse responses (IR) derived from cat auditory nerve fiber data (Carney and Yin, 1988). We will assume that, to a first approximation, the IR functions are comparable with those from owls. For the model simulations, the stimulus was a broadband waveform with a spectrum that ranged from 2 to $8 \mathrm{kHz}$ with an ITD of 0 (i.e., no difference in axonal delay). The sampling rate was $100 \mathrm{kHz}$; therefore, the model resolution was $10 \mathrm{msec}$. Each of two impulse responses, based on the BFs from the ipsilateral and contralateral measurements, was convolved with this stimulus. The two resultant filtered waveforms were cross-correlated (compare Fig. 1), and the delay corresponding to the peak of the cross-correlation function was taken as the predicted ITD (Bonham and Lewis, 1999).

\section{RESULTS}

Data were obtained from a sample of 90 neurons with best frequencies ranging from 3.2 to $7.3 \mathrm{kHz}$. The numbers of neurons used for different analyses varied according to the nature of the data needed.

\section{Frequency-tuning properties during binaural stimulation}

The CF and BF were not significantly different (paired $t$ test) in those cells in which both threshold and isointensity frequency- 

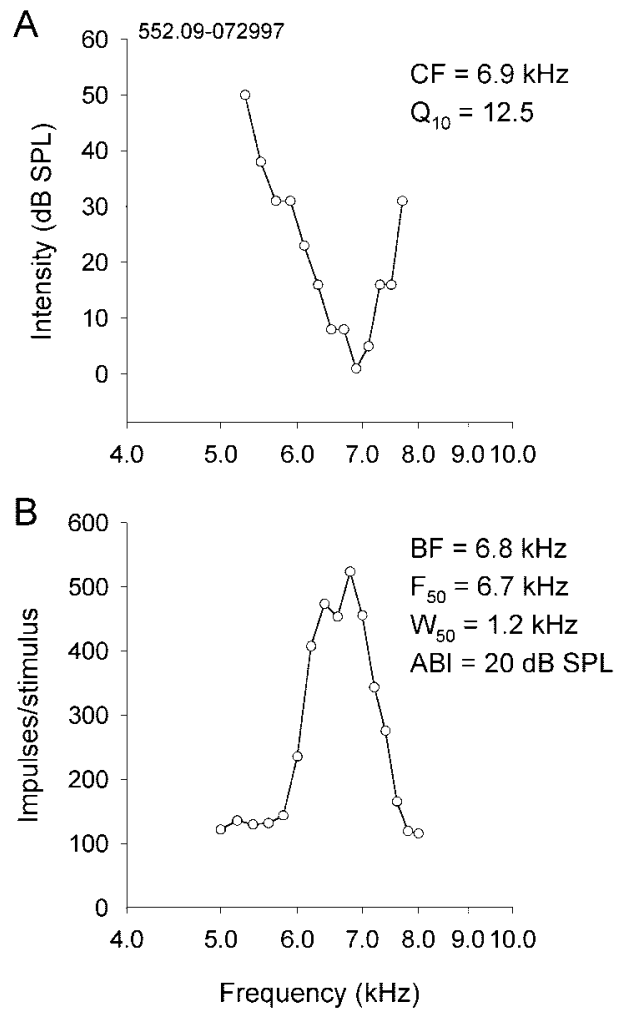

Figure 2. Frequency-tuning curves. Threshold $(A)$ and isointensity $(B)$ tuning curves have similar CFs and BFs. $A B I$, Average binaural intensity (equaling sound levels at two ears divided by 2).
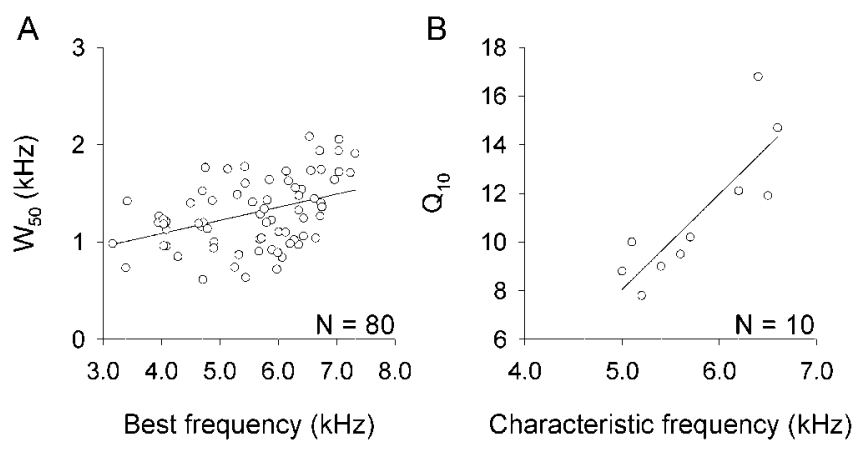

Figure 3. Relationships between preferred frequency and tuning width. $A, \mathrm{~W}_{50}$ plotted against BFs. $B, \mathrm{Q}_{10}$ plotted against CFs. Frequency tuning sharpens as $\mathrm{CF}$ increases (increasing $\mathrm{Q}_{10}$ ).

tuning curves were obtained (10 neurons with CFs in a frequency range of 5-6.6 kHz) with $100 \mathrm{~Hz}$ resolution (Fig. 2). This finding justifies the use of isointensity tuning curves for comparison of the ipsilateral and contralateral inputs to a NL neuron. The tuning widths determined by either the isointensity or the threshold methods were similar. The mean $\mathrm{W}_{50}$ was $1.43 \pm 0.28 \mathrm{kHz}$. $\mathrm{W}_{50}$ increases slightly with $\mathrm{BF}$ (Fig. $3 A$ ). $\mathrm{Q}_{10}$ values had a mean of $11.08 \pm 2.83$ and increased with CF (Fig. $3 B$ ).

NL neurons show phase ambiguity in response to broadband stimuli because of their narrow frequency tuning (Peña and Konishi, 2000). Thus, ITD curves for broadband signals are periodic, with ITD peaks at ITD and ITD $\pm n$ T, where $n$ is an integer and $\mathrm{T}$ is the inverse of the frequency to which the neuron is tuned. $\mathrm{T}$ should be directly correlated with parameters representing frequency tuning. Although $\mathrm{BF}$ and $1 / \mathrm{T}$ values are cor-
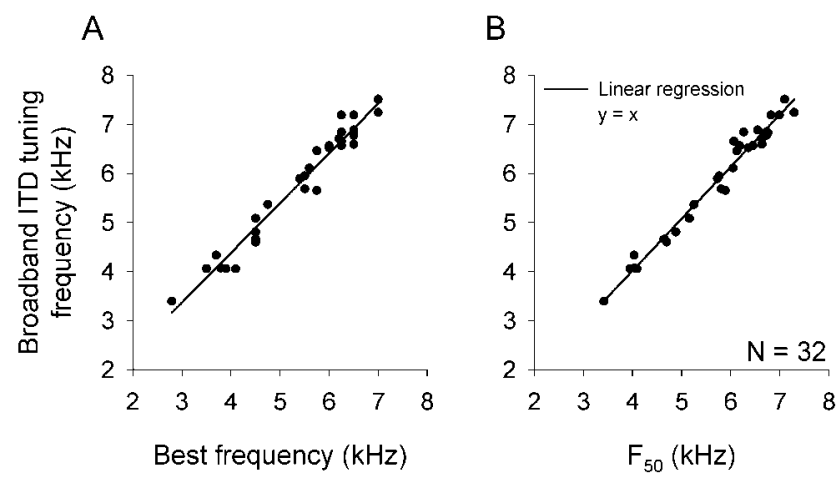

Figure 4. Binaural frequency tuning determined by different methods. Frequencies derived from the period of broadband ITD curves are plotted against BFs $(A)$ and the center frequency $(B)$ obtained for binaural stimuli. The center frequency is a better approximation of the frequency derived from the period of the ITD curve than is BF.

related with each other, $1 / \mathrm{T}$ gave significantly higher values than BF (Fig. 4A). However, the frequencies derived from $1 / \mathrm{T}$ were better correlated with $\mathrm{F}_{50}$ than with BF (Fig. $4 B$ ). Thus, although there is a linear correlation between $\mathrm{BF}$ and the center frequency, we used the latter to compare the bilateral matching in frequency tuning.

\section{Bilateral matching of frequency tuning}

We compared ipsilateral and contralateral frequency tuning in 31 neurons. Interaural frequency mismatches were $<200 \mathrm{~Hz}$ in 23 neurons. In one neuron, the mismatch was $>500 \mathrm{~Hz}$ (Fig. 5A). The mean $\mathrm{W}_{50}$ was $1.01 \pm 0.44 \mathrm{kHz}$ for the ipsilateral side and $1.00 \pm 0.36 \mathrm{kHz}$ for the contralateral side. The mean ipsilateral and contralateral center frequencies were $5.3 \pm 1.2 \mathrm{kHz}$ and $5.4 \pm$ $1.1 \mathrm{kHz}$, respectively, with the center frequency on the contralateral side being significantly higher than that for the ipsilateral side (paired $t$ test, $p=0.02$ ). The mean interaural frequency difference (ipsilateral $\mathrm{F}_{50}$-contralateral $\mathrm{F}_{50}$ ) was $-0.09 \pm 0.2 \mathrm{kHz}$.

The range of frequency mismatches observed and that expected by the stereausis method were different. Using the frequency-dependent latencies of primary auditory fibers in barn owls (Köppl, 1997, their Fig. 7), we calculated the expected frequency mismatches as a function of the best ITD of the neurons for different BFs (Fig. 5B). However, the predicted spectral disparities necessary to produce the best ITD of each neuron (Fig. 5C, open circles) did not agree with the measured disparities (Fig. 5C, filled circles).

Bonham and Lewis (1999) developed a model to predict the ITDs that result from interaural frequency mismatches using the impulse-response model of primary auditory fibers of Carney and Yin (1988). We adapted this model to compute ITDs from the frequency mismatches in our data (see Materials and Methods for details). The correlation between the observed best ITD and the predicted ITD was $r=0.38$ and $r^{2}=0.15\left(t_{(29)}=2.23 ; p<0.05\right)$ (Fig. $5 D$ ). The positive correlation is attributable to three neurons that showed frequency mismatches between 0.4 and $0.5 \mathrm{kHz}$ and were tuned to large ITDs. However, most of the neurons tuned to a large ITD had a small frequency mismatch. In addition, both the observed and predicted ITDs covaried with the best frequency of the neuron ( $r=-0.52$ and $r=-0.34$, respectively), which can account for part of the correlation between the observed and predicted ITD. The first-order partial correlation between the observed ITD and the predicted ITD, with the effect 

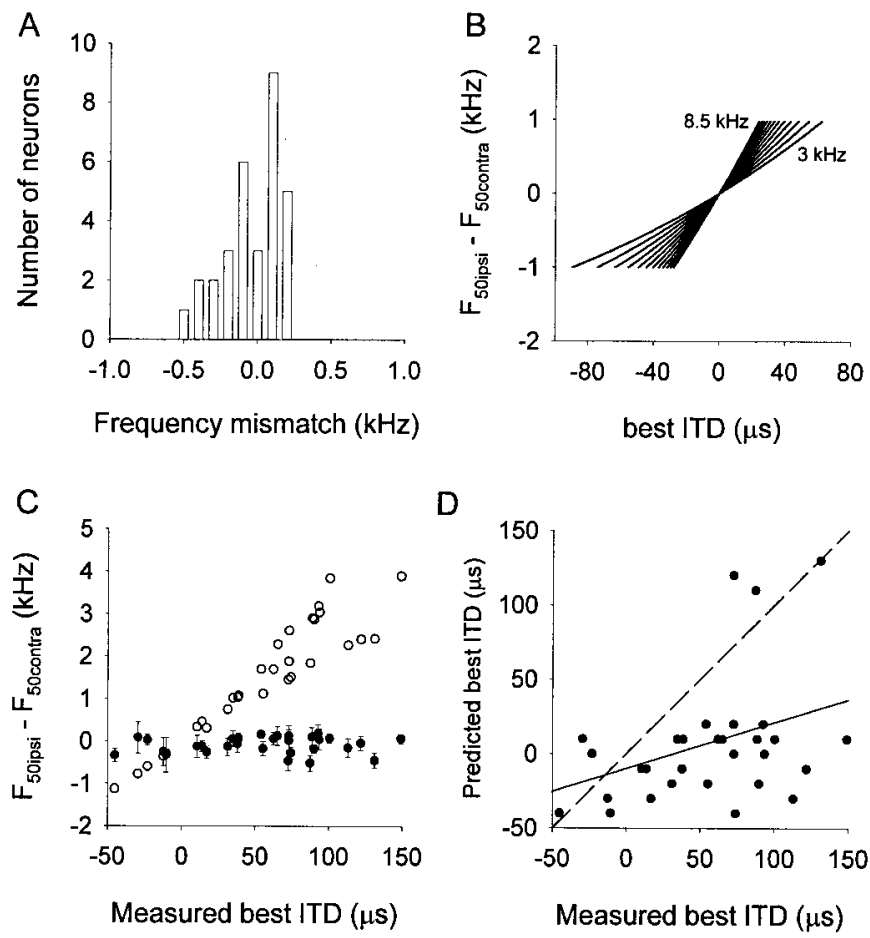

D

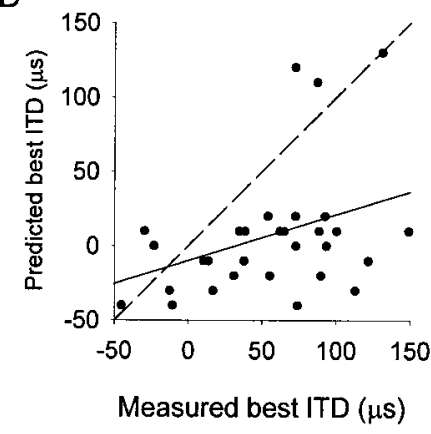

Figure 5. Distribution of frequency mismatches and the preferred ITD. $A$, Most neurons showed a left-right frequency mismatch of $\leq 200 \mathrm{~Hz}$ $(n=21) . B$, Prediction of how the best ITD must change as a function of frequency mismatch and BF using frequency-dependent latencies of primary auditory fibers for the owl (Köppl, 1997). BF was changed in steps of $0.5 \mathrm{kHz}$ and is indicated for the lower $(3 \mathrm{kHz})$ and upper $(8.5 \mathrm{kHz})$ limits. $C$, Differences in the ipsilateral and contralateral center frequencies of $31 \mathrm{NL}$ neurons are plotted against their best ITDs (filled circles). There is no correlation between frequency mismatch and ITD tuning. Error bars indicate SDs. The open circles show the interaural frequency mismatches necessary for encoding ITDs by cochlear delays for each neuron [based on the Bonham-Lewis model adapted for the owl (Köppl, 1997; Bonham and Lewis, 1999]. $D$, The correlation between predicted and observed ITDs showed a correlation of $r=0.38\left(t_{(29)}=2.23 ; p<\right.$ 0.05 ) (solid line). Note that most of the positive correlation is attributable to three neurons. Predicted and observed ITDs also covaried with BF. Removing the effect of BF resulted in a nonsignificant correlation between predicted and observed ITDs (see Results). The dashed line shows a perfect fit between predicted and observed ITDs.

of $\mathrm{BF}$ removed (BF calculated as the average of ipsilateral and contralateral BFs), was closer to 0 and was nonsignificant $(r=$ $\left.0.26, r^{2}=0.07\right)$.

The width of frequency tuning should be greater in binaural than in monaural measurements if the two sides are mismatched in frequency tuning, provided that the width of frequency tuning is similar for the two sides. Thus, the greater the frequency mismatch, the greater the width of binaural frequency tuning. The stereausis theory would predict a correlation between the width of binaural frequency tuning and the magnitude of best ITDs. There was no correlation between them $(r=0.012 ; p=$ 0.934; $n=80$; data not shown).

\section{Axonal delay lines and topographic distribution of ITD}

The ultimate answer to the question of delays other than the axonal delays in the NL must come from direct measurement of these delays and their relationships to ITDs. In some penetrations $(n=13)$, we recorded more than one neuron with the same electrode. We found that the ITD changed system-

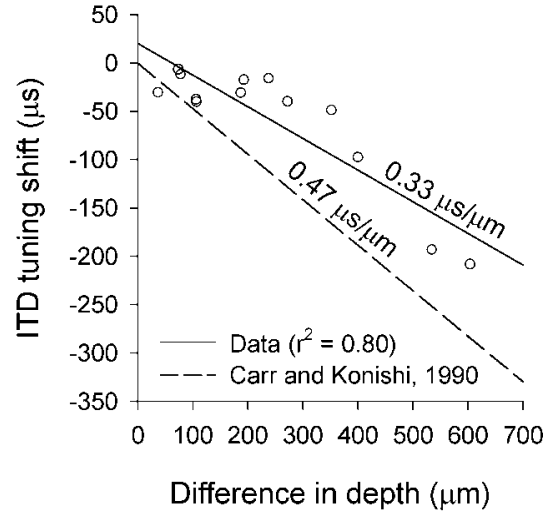

Figure 6. Topographic distribution of ITD tuning in the NL. Data from the present work are indicated by open circles and a regression line (solid line), indicating the change in ITD tuning per micron of depth. For comparison, a regression line (dashed line) from a previous study of magnocellular axons is shown (Carr and Konishi, 1990). Negative ITD tuning shifts indicate changes toward ipsilateral side leading. Differences in depth were calculated by subtracting the smaller depth from the larger depth.

atically with the depth of recording sites along the dorsoventral axis of the NL. This can be explained by an orderly arrangement of delay lines. Axons from the ipsilateral NM enter the $\mathrm{NL}$ from the dorsal side, whereas axons from the contralateral NM enter the NL from the ventral side. Each neuron will be selective to an ITD that exactly cancels out the difference in neural delay of ipsilateral and contralateral inputs. Such a distribution of delay lines, as reported by Carr and Konishi (1990), predicts that the more ventral a neuron is located, the shorter the neural delay for its contralateral input and the longer the delay for its ipsilateral input. Therefore, best ITDs should shift toward the ipsilateral acoustic field with increasingly ventral positions, although ITDs represent only a small portion of the ipsilateral hemisphere. A comparison of delays derived from changes in ITDs in the present work and the delays directly measured by Carr and Konishi (1990) shows some differences (Fig. 6). These differences can be explained by a difference in the penetration angle relative to the dorsal surface of the NL. This nucleus is slanted by $\sim 45^{\circ}$ in the mediolateral direction, with the medial end being deeper from the floor of the fourth ventricle than the lateral end. Carr and Konishi (1990) removed the cerebellum to place the electrode normal to the slanting surface. In the present work, the electrode penetrated the surface at $\sim 45^{\circ}$. We corrected for the difference in the angle of the electrode using trigonometric methods (conversion factor, $1 / \sin 45^{\circ}$ ). The predicted shift in the ITD tuning of our data for a penetration angle of $45^{\circ}$ is $0.47 \mu \mathrm{sec} / \mu \mathrm{m}$, which is identical to the value obtained by Carr and Konishi (1990). Thus, the distribution of axonal delay lines accounts for that of ITDs along the axis normal to the dorsal surface of the NL.

\section{DISCUSSION}

The stereausis theory requires interaural disparities in the cochlear loci from which coincidence detectors receive left and right inputs (Schroeder, 1977; Shamma et al., 1989). Because cochlear loci translate into frequencies, each coincidence detector in this model receives left and right inputs from different frequency 
channels. These spectral disparities determine the ITD to which the stereausis coincidence detectors are tuned.

If this theory were valid, the ITDs to which stereausis coincidence detectors are tuned should be correlated with the magnitude of mismatches in frequency tuning between the two sides. Our results showed first that many neurons (23 of 31) with small or no interaural disparities in frequency tuning had ITDs other than 0 . Second, those neurons with interaural disparities in frequency tuning did not show any correlation between their best ITDs and frequency mismatches. Thus, stereausis predictions are completely different from our findings on the relationships between frequency mismatches and ITDs.

Most NL neurons represent sound directions in the contralateral hemifield (i.e., neurons prefer ITDs in which sounds reach the contralateral ear earlier than the ipsilateral ear). In the stereausis model, this would require that the input frequency of a coincidence detector always be higher (a cochlear locus nearer to the base) on the ipsilateral side than on the contralateral side. We did not see these trends in the owl's nucleus laminaris.

The stereausis model does not consider the effects of sound level on ITD detection. The timing of phase-locked impulses changes with both sound level and frequency in NM and NL neurons (Sullivan and Konishi, 1984; Viete et al., 1997). Shifts in phase increase at a rate of $0.0021 \mu \mathrm{sec} \cdot \mathrm{dB}^{-1} \cdot \mathrm{Hz}^{-1}$ as the stimulating frequency departs from the BF of the neuron (Viete et al., 1997). The direction of phase shifts depends on the sign of frequency differences with respect to the BF of the neuron. Thus, a frequency higher than $\mathrm{BF}$ in one ear and a frequency lower than $\mathrm{BF}$ in the other ear may result in a large shift in the best ITD of the neuron. However, no NL neurons change their best ITDs in response to a variation in sound intensity when it is the same for the two sides (Peña et al., 1996). Therefore, these neurons do not behave like those expected of stereausis coincidence detectors.

Our results showed an ordered sequence of ITDs in the dorsoventral axis of the nucleus laminaris. We measured the rate at which the ITD changes and compared it with that derived from direct measurement of axonal conduction delays (Carr and Konishi, 1990). This agreement indicates that the topographical gradient of ITDs within the NL is entirely attributable to the axonal delay lines. The absence of a correlation between interaural frequency mismatches and ITDs suggests either that the mismatches in frequency do not indicate binaurally unequal cochlear delays or that interaural disparities in cochlear delays do not reach the coincidence detectors.

\section{REFERENCES}

Bonham BH, Lewis ER (1999) Localization by interaural time difference (ITD): effects of interaural frequency mismatch. J Acoust Soc Am 106:281-290.

Carney LH, Yin TCT (1988) Temporal coding of resonances by lowfrequency auditory nerve fibers: single fiber responses and a population model. J Neurophysiol 60:1653-1677.

Carr CE, Konishi M (1988) Axonal delay lines for the time measurement in the owl's brainstem. Proc Natl Acad Sci USA 85:8311-8315.

Carr CE, Konishi M (1990) A circuit for detection of interaural time differences in the brain stem of the barn owl. J Neurosci 10:3227-3246.

Jeffress L (1948) A place theory of sound localization. J Comp Physiol Psych 61:468-486.

Joseph AW, Hyson RL (1993) Coincidence detection by binaural neurons in the chick brain stem. J Neurophysiol 69:1197-1211.

Köppl C (1997) Frequency tuning and spontaneous activity in the auditory nerve and cochlear nucleus magnocellularis of the barn owl Tyto alba. J Neurophysiol 77:364-377.

Peña JL, Konishi M (2000) Cellular mechanisms for resolving phase ambiguity in the owl's inferior colliculus. Proc Natl Acad Sci USA 97:11787-11792.

Peña JL, Viete S, Albeck Y, Konishi M (1996) Tolerance to intense sound of binaural coincidence detection in the owl's nucleus laminaris. J Neurosci 16:7046-7054.

Rose JE, Gross NB, Geisler CD, Hind JE (1966) Some neural mechanisms in the inferior colliculus of the cat which may be relevant to localization of a sound source. J Neurophysiol 29:288-314.

Schroeder MR (1977) New viewpoints in binaural interactions. In: Psychophysics and physiology of hearing (Evans EF, Wilson JP, eds), pp 455-467. New York: Academic.

Shamma SA, Shen N, Gopalaswamy P (1989) Stereausis: binaural processing without neural delays. J Acoust Soc Am 86:989-1006.

Siegel S, Castellan NJ (1988) Nonparametric statistics for the behavioral sciences. New York: McGraw-Hill.

Smith PH, Joris PX, Yin TCT (1992) Projections of physiologically characterized bushy cell axons from the cochlear nucleus of the cat: evidence for delay lines in the medial superior olive. J Comp Neurol 331:245-260.

Sullivan WE, Konishi M (1984) Segregation of stimulus phase and intensity coding in the cochlear nucleus of the barn owl. J Neurosci 4:1787-1799.

Takahashi T, Konishi M (1988) Projections of the cochlear nuclei and nucleus laminaris to the inferior colliculus of the barn owl. J Comp Neurol 274:190-211.

Viete S, Peña JL, Konishi M (1997) Effects of interaural intensity difference on the processing of interaural time difference in the owl's nucleus laminaris. J Neurosci 17:1815-1824.

von Bekesy G (1960) Experiments in hearing. New York: McGraw-Hill.

Yin TCT, Chan JCK (1990) Interaural time sensitivity in the medial superior olive of the cat. J Neurophysiol 64:465-488.

Yin TCT, Kuwada S (1984) Neuronal mechanisms of binaural interaction. In: Dynamic aspects of neocortical function (Edelman GM, Gall WE, Cowan WM, eds), pp 263-313. New York: Wiley.

Young SR, Rubel EW (1986) Embryogenesis of arborization pattern and topography of individual axons in nucleus laminaris of the chicken brain stem. J Comp Neurol 254:425-459. 